\title{
AVALIAÇÃO DA QUALIDADE DA ÁGUA NOS CÓRREGOS FAZZARI E MONJOLINHO NO CAMPUS DA UFSCAR
}

\author{
Germano Henrique Costa Barrilli ${ }^{1}$
}

Odete Rocha $^{2}$

José Valdecir de Lucca $^{3}$

RESUMO: A comunidade bentônica é uma importante indicadora da qualidade ambiental dos ambientes aquáticos, em virtude da sua grande importância para o entendimento da estrutura e funcionamento destes ecossistemas. O uso dos invertebrados bentônicos como bioindicadores na qualidade das águas é amplamente recomendado, pois conseguem refletir as mudanças do ambiente. Neste sentido o objetivo desse trabalho foi avaliar a qualidade da água em 1 ponto de amostragem no Córrego Monjolinho e Fazzari localizados dentro do Campus da UFSCar em São Carlos, utilizando os macroinvertebrados como biondicadores. Para isso, foi aplicado o índice BMWP e foi calculada a relação $\mathrm{O} / \mathrm{O}+\mathrm{C}$. As coletas foram realizadas em março e abril de 2012. Os organismos foram coletados com um amostrador do tipo Surber (análise quantitativa) e os espécimes preservados em álcool 70\%. Em seguida foram identificados com auxílio de estereomicroscópio óptico e bibliografia especializada. № córrego do Fazzari foram registrados 173 indivíduos, destes 98 pertence a família Chironomidae. Já no Monjolinho foram registrados a ocorrência de 456 indivíduos sendo 354 representantes da Classe Oligochaeta. Os valores obtidos relação $\mathrm{O} / \mathrm{O}+\mathrm{C}$ e os "scores" do índice BMWP sugerem que o Monjolinho encontra-se impactado enquanto que 0 Fazzari preservado. Os resultados da pesquisa auxiliam em futuros estudos nesses locais como sendo uma ferramenta para o monitoramento desses córregos dentro do Campus da UFSCar.

Palavras-chave: BMWP, macroinvertebrados bentônicos. Córrego Fazzari e Monjolnho

\footnotetext{
1 Biólogo, Universidade Federal de São Carlos. germanohcb@msn.com

${ }^{2}$ Biólogo, Universidade Federal de São Carlos. doro@ufscar.br

${ }^{3}$ Biólogo, Universidade Federal de São Carlos. E-mail. julucca@bol.com.br
} 


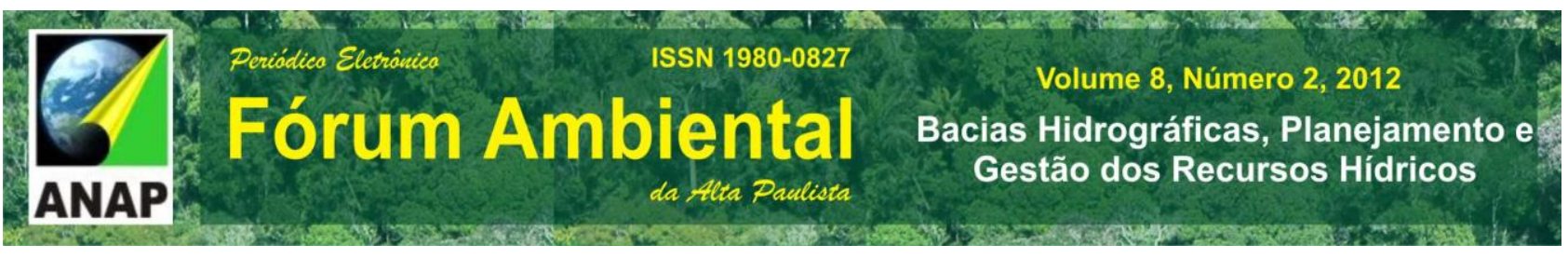

\section{INTRODUÇÃO}

Atividades antrópicas como agricultura, pecuária, mineração e o uso da terra para o estabelecimento da população, contribuem para o despejo de dejetos contaminados e para alterações no habitat aquático (QUEIROZ et. al, 2008).

Atualmente, um dos maiores problemas a serem solucionados na área de ciências ambientais é a restauração de habitats e geralmente com vistas ao restabelecimento das funções e processos que são influenciados por fatores físicos, químicos e biológicos, envolvendo os ecossistemas aquáticos, terrestres e ripários associados às bacias hidrográficas (KAUFFMAN et al., 1997). Por ser um tema de grande importância, os projetos de recuperação vem desempenhando um papel cada vez maior no gerenciamento e manejo ambiental em decisões políticas (PALMER, 2004). Esses projetos podem tornar-se bem sucedidos se forem efetivos na recuperação de espécies, melhoria na qualidade das águas continentais e aumento da diversidade de habitats que facilitem a recolonização por espécies nativas (BERNHARDT et al., 2005).

Uma das ferramentas para se avaliar a qualidade ambiental é o uso de organismos bioindicadores. A utilização desses organismos vivos como ferramentas para 0 monitoramento de ambientes aquáticos serve para avaliar alterações ocorridas em locais que, geralmente, são atribuídas a ações antrópicas (BUSS et al., 2003). Nos sistemas aquáticos os insetos são apropriados para tal finalidade por serem diversos, apresentarem ciclo rápido e serem facilmente amostrados (SILVEIRA NETO et al.,1995) Nesse contexto, comunidades bentônicas podem ser utilizadas como indicadores de qualidade de ambientes aquáticos, onde sua estabilidade e permanência são determinadas pelas condições do meio em que vivem (MENEGATT et al, 2007).

A comunidade de macroinvertebrados possui grande diversidade de espécies, diversas formas e modo de vida, e podem ser encontrados em corredeiras, riachos, rios, lagos e represas (SILVEIRA et al.; 2004). Apresentam características importantes como: locomoção reduzida; amostragem simples; são organismos de fácil identificação até o 


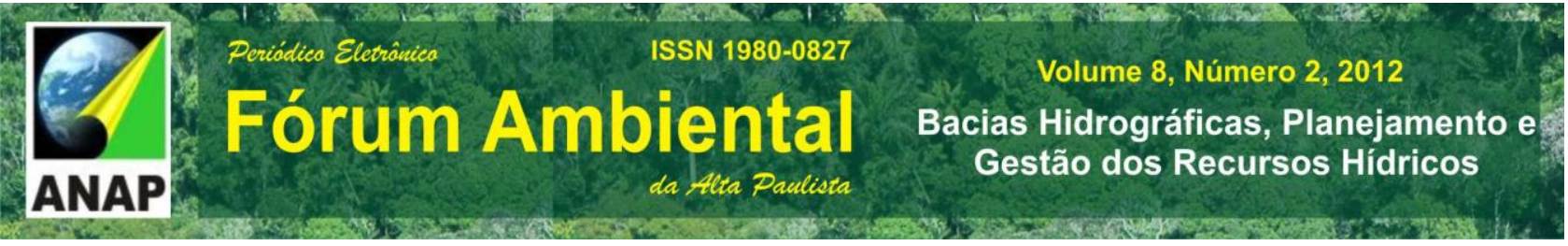

nível de família; respondem rapidamente a distúrbios; além de possuírem vários táxons que respondem de diferentes formas aos diversos tipos e níveis de poluição (BUSS et al., 2003). Essas características têm contribuído para o uso cada vez mais frequente desses organismos como bioindicadores em várias partes do mundo, especialmente nos Estados Unidos, Austrália, e países europeus (SURIANO et al., 2010).

CAPITOLI e BEMVENUTTI (2004), afirmam que alterações na diversidade de macroinvertebrados bentônicos podem estar relacionadas a distúrbios no ambiente e consequentemente, dependendo do grau de alteração, o desenvolvimento da comunidade é afetado, restando poucas espécies que são tolerantes a esses eventos. Nesse sentido, a família Chironomidae e toda classe Oligochaeta são denominados organismos tolerantes, podendo viver em condições de anóxia e não apresentam nenhum tipo de exigência quanto à diversidade de hábitats e microhábitats (GOULART e CALLISTO, 2003). Para a avaliação da qualidade da água através da utilização dos macroinvertebrados bentônicos vários índices têm sido propostos. Entre eles, um dos mais utilizados em todo o mundo é o BMWP "Biological Monitoring Working Party score System". Este índice criado em 1976 na Grã Bretanha, com o intuito de monitorar a biota aquática e, atualmente, tem sido utilizado com macroinvertebrados bentônicos, identificados até nível de família, com o objetivo de se avaliar a qualidade da água (MONTEIRO et. al, 2008). Deste modo, famílias mais sensíveis aos impactos recebem pontuações mais elevedas, e as mais resistentes recebem pontuação baixas, de forma a gerar um gradiente de organismos sensíveis à tolerantes. Quanto maior o "score" obtido menor a poluição do ambiente aquático (BUSS et al., 2003)

O objetivo do presente trabalho é a utilização dos macroinvertebrados bentônicos através da aplicação do índice adaptado BMWP (ALBA TERCEDOR E SÁNCHEZ ORTEGA, 1988; ALBA TERCEDOR, 2000) e da relação Oligochaeta/Chironomidae proposta por WIEDERHOLM (1980) para avaliação preliminar da qualidade da água em 2 córregos localizados dentro do campus da UFSCar em São Carlos.

\section{2. ÁREA DE ESTUDO}




\section{Rio Monjolinho}

A bacia no rio Monjolinho que abrange uma área de aproximadamente $275 \mathrm{~km} 2$, com sua maior parte contida no município de São Carlos-SP (SÉ, 1992), tem como principal característica o desenvolvimento urbano de São Carlos e os impactos de suas atividades e possui uma extensão de 43,25 Km (SÉ, 1992).

Formado pela barragem do córrego Monjolinho, o reservatório possui 4,96 ha e está localizado nas coordenadas $47^{\circ} 54^{\prime} \mathrm{W}-22^{\circ} 00^{\prime} \mathrm{S}$ no campus da Universidade Federal de São Carlos, município de São Carlos, SP. Apresenta profundidade baixa, sendo a média 1,70 metros e a máxima 3,00 metros. A área de inundação é de 47.157 metros quadrados e o seu volume é de 73.251 metros cúbicos. O Ponto de coleta está localizado nas coordenadas $21^{\circ} 59^{\prime} 05.0^{\prime \prime} \mathrm{S}$ e $45^{\circ} 53^{\prime} 06.1^{\prime \prime} \mathrm{W}$.

\section{Córrego Fazzari}

O segundo ponto de coleta também localizado dentro do Campus da UFSCar encontra-se em uma área não urbanizada, próximo a sua nascente onde foi demarcado com as coordenadas geográficas $21^{\circ} 59^{\prime} 05.0^{\prime \prime} \mathrm{S}$ e $45^{\circ} 53^{\prime} 06.1^{\prime \prime} \mathrm{W}, 794 \mathrm{~m}$ de altitude, este ponto situa-se dentro de uma área de preservação do Campus tendo no seu entorno a vegetação de cerrado. O Córrego do Fazzari, de primeira ordem possui pequenas dimensões com profundidade máxima de $0,60 \mathrm{~m}$ e largura máxima de 1,50m, apresenta vegetação ripária e mata ciliar bem preservada (SIQUEIRA \& TRIVINHO-STRIXINO, 2005).

A represa do Fazzari está localizada a 500 metros da nascente e foi construída em 1978 com o objetivo de fornecer água para irrigação da horta e do pomar da Universidade, localizados a mercê de suas margens (ALBUQUERQUE, 1990), porém hoje não possui essa finalidade. Após seu represamento parte de sua mata ciliar ficou parcialmente submersa, possuindo ótimo afloramento de água subterrânea e água de boa qualidade, adequada para abastecimento e demais usos (TRIVINHO-STRIXINO \& STRIXINO, 1998). O córrego do Fazzari juntamente com a represa são afluentes do Rio 
Monjolinho que integra bacia hidrográfica a do rio Jacaré-Guaçú (ROQUE \& TRIVINHOSTRIXINO, 2001).

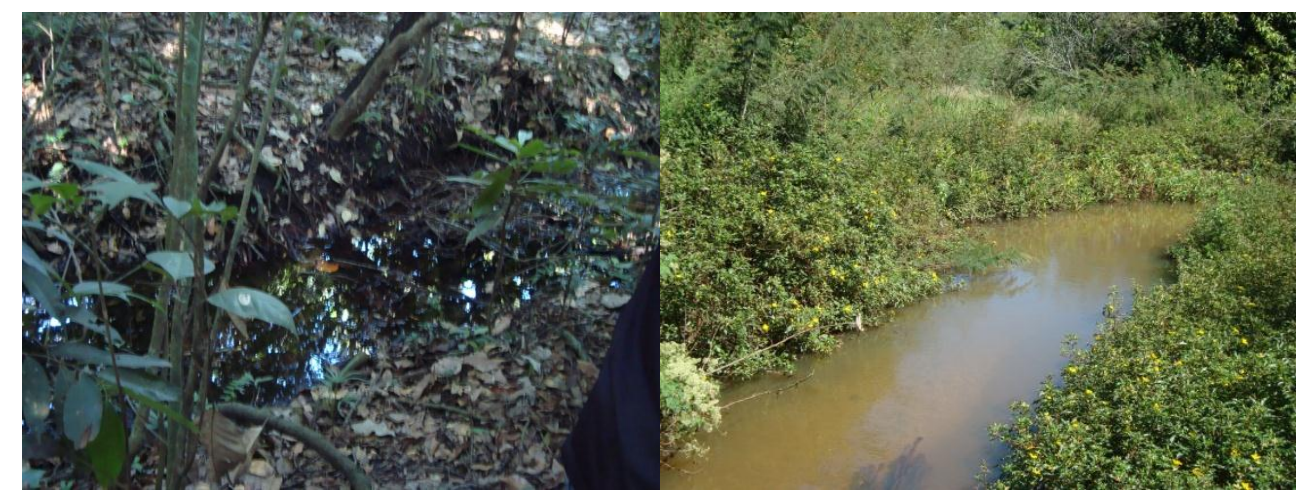

Figura 1 - Córrego Fazzari (Esquerda) e Corrégo Monjolinho (Direita)

\section{MATERIAIS E MÉTODOS}

\section{Coleta e identificação dos macroinvertebrados bentônicos}

As coletas dos organismos bentônicos foram realizadas em março e abril de 2012 com amostrador Surber com tela 250 micra de abertura de malha e área amostral 0,3m². As amostragens foram realizadas em tréplicas.

O material coletado foi acondicionado em recipientes plásticos e transportado para o laboratório, onde foi lavado em água corrente sobre peneira com malha de 0,21 $\mathrm{mm}$. Os espécimes retidos na peneira foram separados em bandeja transiluminada e fixados em álcool a $70 \%$ até o momento da identificação.

Os macroinvertebrados foram identificados, quando possível, até o nível taxonômico de Família sob microscópio estereoscópico, com o auxílio de literatura especializada: McCafferty,1981; Pérez, 1988; Brinkhurst \& Marchese, 1989; TrivinhoStrixino \& Strixino, 1995; Merritt \& Cummins, 1996; Também foram realizadas medidas "in situ" na água do potencial hidrogeniônico $(\mathrm{pH})$, condutividade elétrica, oxigênio dissolvido e temperatura. 


\section{Tratamento dos dados}

Indice BMWP (Biological Monitoring Working Party score System)

Foi atribuída uma pontuação ("Score") para cada família identificada (Tabela 1), através do índice BMWP, conforme o modelo modificado proposto por Alba-Tercedor e Sánchez-Ortega, (1988);

\section{Tabela 1 - Índice BMWP - ( ALBA-TERCEDOR \& SÁNCHEZ-ORTEGA, 1988; ALBA- TERCEDOR, 2000)}

\begin{tabular}{|c|c|}
\hline FAMILIAS & SCORE \\
\hline $\begin{array}{l}\text { Siphlonuridae, Heptageniidae, Leptophlebiidae, Potamanthidae, } \\
\text { Taeniopterygidae, Leuctridae, Capniidae, Perlodidae, Perlidae } \\
\text { Chloroperlidae, Aphelocheirida, Phryganeidae, Molannidae, Beraeidae, Odontoceridae, } \\
\text { Leptoceridae, Goeridae, Lepidostomatidae, Brachycentridae, Sericostomatidae } \\
\text { Athericidae, Blephariceridae }\end{array}$ & 10 \\
\hline $\begin{array}{l}\text { Lestidae, Calopterygidae, Gomphidae, Cordulegasteridae, Aeshnidae } \\
\text { Corduliidae, Libellulidae, Psychomyiidae, Philopotamidae, Glossosomatidae }\end{array}$ & 8 \\
\hline $\begin{array}{l}\text { Ephemerellidae, Prosopistomatidae, Nemouridae, Rhyacophilidae, Polycentropodidae, } \\
\text { Limnephilidae, Ecnomidae }\end{array}$ & 7 \\
\hline $\begin{array}{l}\text { Neritidae, Viviparidae, Ancylidae, Thiaridae, Hydroptilidae, Unionidae, Corophiidae, } \\
\text { Gammaridae, Atyidae, Platycnemididae, Coenagrionidae }\end{array}$ & 6 \\
\hline $\begin{array}{l}\text { Oligoneuriidae, Polymitarcidae, Dryopidae, Elmidae, Helophoridae, Hydrochidae, } \\
\text { Hydraenidae, Clambidae, Hydropsychidae, Tipulidae, Simuliidae, Planariidae, } \\
\text { Dendrocoelidae, Dugesiidae }\end{array}$ & 5 \\
\hline $\begin{array}{l}\text { Baetidae, Caenidae, Haliplidae, Curculionidae, Chrysomelidae, Tabanidae, Stratiomyidae, } \\
\text { Empididae, Dolichopodidae, Dixidae, Ceratopogonidae, Anthomyidae, Limoniidae, } \\
\text { Psychodidae, Sciomyzidae, Rhagionidae, Sialidae, Piscicolidae, Hidracarina }\end{array}$ & 4 \\
\hline $\begin{array}{l}\text { Mesoveliidae, Hydrometridae, Gerridae, Nepidae, Naucoridae, Pleidae, Veliidae } \\
\text { Notonectidae, Corixidae, Helodidae, Hydrophilidae, Hygrobiidae, Dytiscidae, Gyrinidae, } \\
\text { Valvatidae, Hydrobiidae, Lymnaeidae, Physidae, Planorbidae, }\end{array}$ & 3 \\
\hline $\begin{array}{l}\text { Bithyniidae, Bythinellidae, Sphaeridae, Glossiphoniidae, Hirudidae, } \\
\text { Asellidae, Ostracoda }\end{array}$ & \\
\hline Chironomidae, Culicidae, Ephydridae, Thaumaleidae & 2 \\
\hline Oligochaeta (toda a classe), Syrphic & 1 \\
\hline
\end{tabular}


Conforme a soma dos "Scores" das famílias, cada ambiente foi classificado de acordo com os intervalos de valores proposto pelo índice BMWP (tabela 2)

Tabela 2 - Valores de referência do índice BMWP

\begin{tabular}{clclc}
\hline CLASSE & QUALIDADE & VALOR & SIGNIFICADO & COR \\
\hline I & BOA & $>150$ & Água muito limpas & AZUL \\
& & $101-120$ & Águas não contaminadas & \\
II & ACEITÁVEL & $61-100$ & $\begin{array}{l}\text { Águas com alguns efeitos de } \\
\text { contaminação evidente. }\end{array}$ & VERDE \\
III & DUVIDOSA & $36-60$ & Águas poluída & AMARELO \\
IV & CRÍTICA & $16-35$ & Águas muito poluída & LARANJA \\
V & $\begin{array}{l}\text { MUITO } \\
\text { CRÍTICA }\end{array}$ & $<15$ & Águas fortemente poluída & VERMELHO \\
\hline
\end{tabular}

\section{Relação Oligochaeta/ Chironomidae}

A relação Oligochaeta/Chironomidae proposta por Wiederholm (1980) aponta um gradiente de eutrofização no ambiente aquático pela seguinte fórmula:

$$
\mathrm{O} /(\mathrm{O}+\mathrm{C}) \text { onde, }
$$

O - representa o número de indivíduos da Classe Oligochaeta

C - representa o número de indivíduos da família Chironomidae

Quanto mais próximo de 1, maior é o grau de eutrofização/poluição do ambiente. 


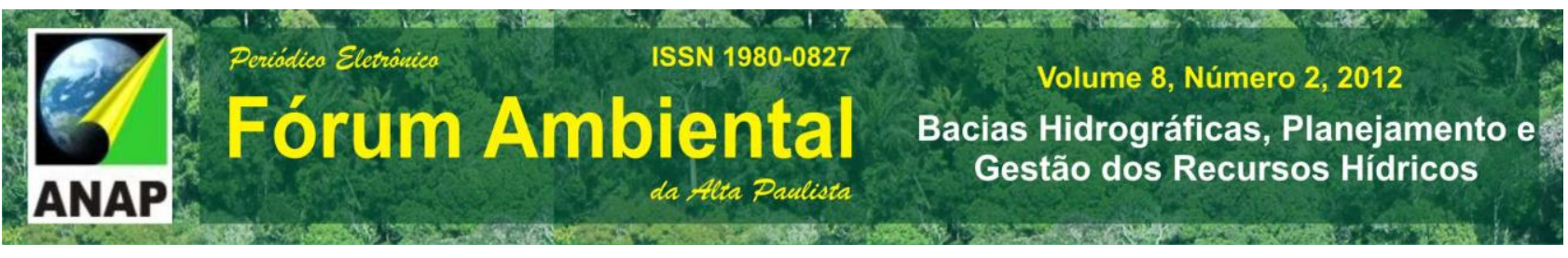

\section{RESULTADOS E DISCUSSÀO}

No córrego do Fazzari, em março, foi registrado um total de 124 indivíduos enquanto que em abril foi de apenas 49 indivíduos (Tabela 3). Nos 2 períodos amostrados, os representantes da família Chironomidae foram os mais representativos numericamente.

No Monjolinho foi registrado em março a ocorrência de 370 espécimes enquanto que em abril foi registrado apenas 96 (Tabela 3). Em ambos os períodos, os anelídeos da Classe Oligochaeta foram os mais abundantes.

Em decorrência de impactos causados pela retirada da mata ciliar, o uso e ocupação do solo como urbanização e atividades inerentes agricultura e pecuária, o rio Monjolinho se encontra impactado. Esses impactos refletem no desaparecimento de espécies de macroinvertebrados bentônicos sensíveis e promovem a dominância de espécies resistentes como os grupos Oligochaeta e alguns Chironomidae. Segundo GOULART E CALLISTO (2003), grupos de macroinvertebrados bentônicos ditos como resistentes conseguem viver em condições de severa falta de oxigênio e não são exigentes quanto à diversidade de habitats e microhabitats, podendo ser encontrados tanto em ambientes límpidos quanto poluídos. De acordo com Wetzel, 1983, os Oligochaeta são bons indicadores da qualidade da água. No presente trabalho foi registrado elevada densidade de Oligochaeta no Monjolinho notadamente em março de 2012. Segundo Milbrink, (1994) a presença desses anelídeos tem sido reportada em ambientes organicamente poluídos.

Já o córrego Fazzari, apresenta uma área de entorno com vegetação mais estruturada, portanto se encontra mais preservado. Apesar da dominância de grupos mais resistentes (Oligochaeta e Chironomidae), esse ambiente apresentou maior riqueza taxonômica e, dentre os espécimes, representantes de tricópteros mais sensíveis à poluição como os Calamoceratidae e Leptoceridae. As larvas da família Chironomidae são frequentemente consideradas como indicadoras de poluição. Porém é comum que rios não poluídos tenham grande abundância dessa família (McCULLOCH, 1986; 
PINDER,1986) fazendo com que essa família seja comumente encontrada nos mais diversos tipos de ambientes aquáticos. Essas informações corroboram com o observado no córrego do Fazzari que apesar de não apresentar nenhum tipo de impacto antrópico foi registrado elevada densidade de Chironomidae. Grupos sensíveis requerem alta diversidade de habitats e microhabitats e uma oxigenação do ambiente aquático, ou seja, um alto grau de qualidade do ambiente (GOULART E CALLISTO, 2003). Nesse contexto, o córrego Fazzari pode ser enquadrado em um ambiente propício para o desenvolvimento da fauna de organismos bentônicos com maior sensibilidade.

Tabela 3 - Composição taxonômica e abundância absoluta (AA) dos os córregos amostrados nos meses de Março e Abril de 2012 no campus da UFSCar - SP

\begin{tabular}{ccccc} 
& \multicolumn{2}{c}{ MARÇO/2012 } & \multicolumn{2}{c}{ ABRIL/2012 } \\
TAXÓNS & FAZARI & MONJOLINHO & FAZZARI & MONJOLINHO \\
Anellida - Hirudinea & AA & AA & AA & AA \\
Oligochaeta & 0 & 9 & 2 & 15 \\
Coleoptera - Hidrophilidae & 15 & 324 & 1 & 30 \\
Dysticidae & 0 & 17 & 1 & 4 \\
Elmidae & 5 & 0 & 0 & 0 \\
Diptera - Ceratopogonidae & 3 & 0 & 5 & 0 \\
Chironomidae & 11 & 0 & 0 & 0 \\
Tipulidae & 65 & 14 & 33 & 30 \\
Ephemeroptera - Baetidae & 11 & 0 & 0 & 0 \\
Hemiptera - Helotrephidae & 1 & 0 & 1 & 0 \\
Nematoda & 0 & 0 & 1 & 0 \\
Odonata - Aeshnidae & 1 & 0 & 0 & 0 \\
Calopterygidae & 0 & 0 & 0 & 1 \\
Coenagrionidae & 0 & 0 & 2 & 6 \\
Gripopterygidae & 2 & 6 & 0 & 0 \\
Libellulidae & 0 & 0 & 1 & 0 \\
Trichoptera - Calamoceratidae & 6 & 0 & 0 & 10 \\
Leptoceridae & 0 & 0 & 1 & 0 \\
& 4 & 0 & 1 & 0 \\
\hline
\end{tabular}


O índice BMWP e a relação Oligochaeta/Chironomidae foram aplicados para cada ambiente. Para isso acumulou-se as família em cada ambiente conforme a tabela 4. De acordo com a pontuação gerada pelo índice BMWP, o córrego Monjolinho se encontra em Classe IV, portanto crítico, caracterizando suas águas como muito poluída. Já o córrego Fazzari em nível aceitável (classe II). Já pelo método proposto por Wiederholm (1980), onde quanto mais próximo do valor 1 (resultado do valor entre a relação Oligochaeta/ Chironomidae) mais poluído é o ambiente, o córrego Monjolinho foi valorado como sendo o mais impactado $(\mathrm{O} / \mathrm{O}+\mathrm{C}=0,89)$ em relação ao Fazzari que registrou um valor significantemente inferior dessa relação $(\mathrm{O}+\mathrm{O} / \mathrm{C}=0,14)$. $\mathrm{O}$ índice $\mathrm{BMWP}$ e a relação $\mathrm{O} / \mathrm{O}+\mathrm{C}$ evidenciaram que o Monjolinho encontra-se impactado enquanto que o Fazzari mais preservado.

Tabela 4 - Valores de abundância, "Scores" BMWP e Relação Oligochaeta/Chironomidae nos córregos Monjolinho e Fazzari

\begin{tabular}{lcccc}
\multicolumn{1}{c}{ TAXÓNS } & FAZZARI & BMWP & MONJOLINHO & BMWP \\
Anellida - Hirudinea & 2 & 3 & 24 & 3 \\
Anellida - Oligochaeta & 16 & 1 & 354 & 1 \\
Coleoptera - Hidrophilidae & 1 & 3 & 21 & 3 \\
Coleoptera - Dysticidae & 5 & 3 & 0 & \\
Coleoptera - Elmidae & 8 & 5 & 0 & 2 \\
Diptera - Ceratopogonidae & 11 & 4 & 0 & \\
Diptera - Chironomidae & 98 & 2 & 44 & \\
Diptera - Tipulidae & 11 & 5 & 0 & \\
Ephemeroptera - Baetidae & 2 & 4 & 0 & 8 \\
Hemiptera - Helotrephidae & 1 & & 0 & 6 \\
Nematoda & 0 & & 0 & \\
Odonata - Aeshnidae & 0 & & 1 & \\
Odonata - Calopterygidae & 2 & 8 & 6 & 8 \\
Odonata - Coenagrionidae & 2 & 6 & 6 & \\
Odonata - Gripopterygidae & 1 & & 0 & \\
Odonata - Libellulidae & 6 & 8 & 10 & \\
Trichoptera - Calamoceratidae & 1 & & 0 & \\
Trichoptera - Leptoceridae & 5 & 10 & 0 & \\
TOTAL & $\mathbf{1 7 2}$ & $\mathbf{6 2}$ & $\mathbf{4 6 6}$ & \\
Relação O/(O+C) & $\mathbf{0 . 1 4}$ & & $\mathbf{0 . 8 9}$ &
\end{tabular}


Efeitos sobre a comunidade macrozoobentônica foram avaliados por MENDES (1998) no Rio Monjolinho desde a nascente até a foz, analisando várias ocupações, desde áreas preservadas até áreas com ocupação urbana e agrícola. $\mathrm{O}$ autor conclui que há um impacto sobre a macrozoofauna ao longo do rio, reforçando que os impactos podem ser sentidos por esses organismos em virtude de ações antrópicas. Esse aspecto corrobora com os dados encontrados no presente estudo, uma vez que a riqueza de táxons no rio Monjolinho foi menor em relação ao Fazzari.

Alguns trabalhos reforçam a importância da preservação na mata ciliar na manutenção da fauna macrobentônica, segundo os autores FERREIRA-PERUQUETI E FONSECA-GESSNER, (2003); BENSTEAD e PRINGLE, (2004), afirmam que áreas que possuem mata ciliar é possível observar uma fauna mais rica e heterogênea em relação á áreas sem essa cobertura vegetal.

\section{CONCLUSÃO}

Oligochaeta obteve maior representatividade do córrego Monjolinho, enquanto Chironomidae foi mais abundante no córrego Fazzari.

O índice BMWP classificou como "Crítico" o córrego Monjolinho e "Aceitável" o córrego Fazzari.

A relação Oligochaeta/Chironomidae apontou o córrego Monjolinho como mais poluído do que o Fazzari.

O córrego Fazzari registrou uma maior diversidade de táxons inclusive com a ocorrência de espécies sensíveis (Trichoptera) e, portanto uma fauna mais heterogênea que o Monjolinho. 


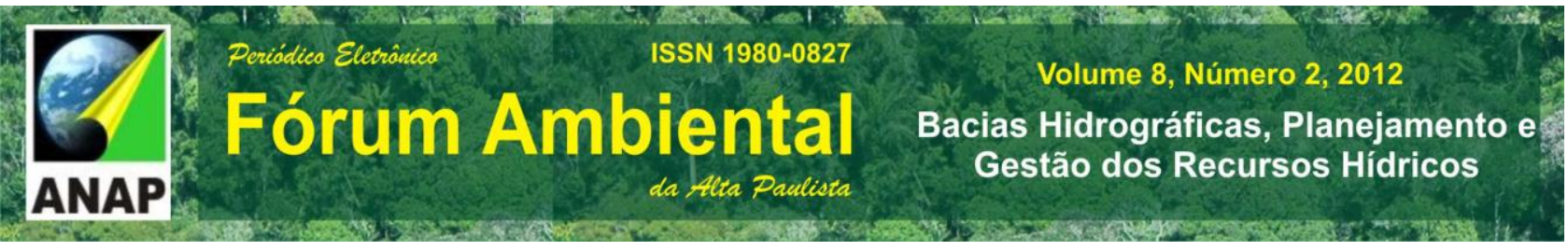

Apesar de ter sido realizado apenas duas campanhas, a utilização dos macroinvertebrados como bioindicadores através da aplicação do índice BMWP e a Relação Oigochaeta/Chironomidae foram eficientes na avaliação da qualidade da água nos 2 sistemas estudados.

\section{REFERÊNCIAS}

ALBA-TERCEDOR, J., SÁNCHEZ-ORTEGA, A. Un método rápido y simples para evaluar la calidad biológica de las aguas corrientes basado en el Hellawell (1978). Limnética 4,51-56., 1988.

ALBA-TERCEDOR, J. (BMWP', un adattamento spagnolo del British Biological Monitoring Working Party (BMWP) Score System. Biol. Amb., 14 (n.‥ 2) pp.: 65-67, 2000

ALBUQUERQUE, L.B. Entomofauna aquatic do litoral de dois reservatórios da região de São Carlos - SP. São Carlos - Sp, UFSCar, 94 p. Dissertação (Mestrado), 1990.

BENSTEAD, J.P. AND PRINGLE, C.M. Deforestation alters the resource base and biomass of endemic stream insects in eastern Madagascar. Freshwater Biol., 49, 490501,2004

BERNHARDT E. S.; PALMER, M.; ALLAN, J. D.; ALEXANDER, G.; BARNAS, K.; BROOKS, S.. Ecology - Synthesizing US River Restoration Efforts. Science, v. 308, p.636-637, 2005

BRINKHURST, R. O. e MARCHESE, M. R. Guia de la identificacion de Oligoquetos acuaticos continentales de Sud y Centroamerica. Associación Ciencias Naturales del Litoral. Argentina. Colección Climax (5), 207p., 1989

BUSS, F. D; BAPTISTA, D. F; NESSIMIAN, J.L. Bases conceituais para a aplicação de biomonitoramento em programas de avaliação da qualidade da água de rios. Cad. Saúde Publica. Rio de Janeiro, v. 19, nº 2, p. 465-473, 2003.

CAPITOLI, R.R., BEMVENUTTI, C. E. Distribuição batimétrica e variações de diversidade de macroinvertebrados bentônicos da plataforma continental e talude superior no extremo sul do Brasil. Atlântica, Rio Grande, nº 26: 27-43, 2004.

FERREIRA-PERUQUETTI, e P. FONSECA-GESSNER, A.A. Odonata community on natural areas of Cerrado and monoculture of northeastern São Paulo State, Brazil: relationship between land use and richness. Revta. bras. Zool., 20, 219-224, 2003 


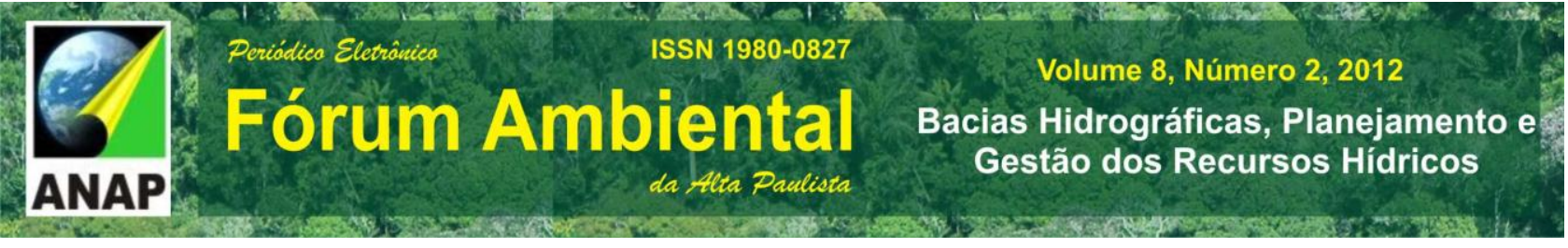

GOULART, M. e CALLISTO, M. Bioindicadores de qualidade de água como ferramenta em estudos de impacto ambiental. Revista da FAPAM, ano 2, $n^{0} 1,2003$

KAUFFMAN, J. B.; BESCHTA, R. L.; OTTING N.; LYTJEN, D. An ecological Perspective of Riparian and Stream Restoration in the Western United States. Fisheries, v. 22, p. 1224, 1997

McCULLOCH, D. L. Benthic macroinvertebrate distributions in the riffle-pool communities of two east Texas streams. Hydrobiologia, v. 135, p. $61-70,1986$

MENDES, A.J.S. Avaliação dos impactos sobre a comunidade macrozoobentônica, no córrego do Monjolinho (São Carlos, SP). Dissertação de Mestrado. Escola de Engenharia de São Carlos, Universidade de São Paulo, São Carlos, 1998

MERRITT, R.W.; CUMMINS, K.W. (Org.). An introduction to the aquatic insects of North America. 3aㅗ ed. Dubuque: Kendall/Hunt Publishers, 862 p, 1996.

MENEGATT, C. et al. Diversidade de macroinvertebrados bentônicos de um rio da região Alto Uruguai - RS. Sociedade de Ecologia do Brasil. MG, 2007

MILBRINK, G. Oligochaetes and water pollution in two deep Norwegian lakes. Hydrobiologia 278:213-222, 1994

MONTEIRO, T.R. et al. Biomonitoramento da qualidade de água utilizando macroinvertebrados bentônicos. Oecol. Bras., 12 (3): 553-563, 2008

PALMER, M.; BERNHARDT E. S.; CHORNESKY, E.; COLLINS, S.; DOBSON, A.; DUKE, C.2004. Ecology for a Crowded Planet. Science, v.304, p. 1251-1252.

PÉREZ, G. A. R. Guía para el estudio de los macroinvertebrados acuáticos del Departamento de Antioquia. Bogotá: Fondo Fen Colômbia, Colciencias/Universidad de Antioquia, 1988.

PINDER, L. C. V. Biology of Freshwater Chironomidae. Annu. Rev. Entomol., v. 31, p. 1 23, 1986.

QUEIROZ, J. F. et al. 2008. Organismos bentônicos bioindicadores da qualidade de água. EMBRAPA Meio Ambiente. 91 p. Jaguariúna.

ROQUE, F. O.; TRIVINHO-STRIXINO, S. Benthic macroinvertebrates in mesohabitats of different spatial dimensions in a first order stream (São Carlos-SP).Acta Limnologica Brasiliensia, v. 13, p. 69-77, 2001.

SÉ, J. A. S. O Rio Monjolinho e sua Bacia Hidrográfica como Integradores de Sistemas Ecológicos. 381 p. Dissertação (Mestrado). Escola de Engenharia de São Carlos, Universidade de São Paulo, São Carlos, SP, 1992. 
SILVEIRA NETO, S.; MONTEIRO, R. C.; ZUCCHI, R. A.; MORAES, R. C. B. Uso da análise faunística de insetos na avaliação do impacto ambiental. Scientia. Agricola, Piracicaba, v. 52, n. 1, p. 09-15, 1995.

SILVEIRA, M.P. Aplicação do biomonitoramento para avaliação da qualidade da água em rios. São Paulo: EMBRAPA, 68p. (Documentos, 36), 2004.

SIQUEIRA T, TRIVINHO-STRIXINO S. Diversidade de Chironomidae (Diptera) em dois córregos de baixa ordem na região central do Estado de São Paulo, através da coleta de exúvias de pupa. Rev Bras Entomol 49: 531-534., 2005.

SURIANO, M. T.; FONSECA-GESSNER, A. A.; ROQUE, F. O.; FROEHLICH, C. G. Choice of macroinvertebrate metrics to evaluate stream conditions in Atlantic Forest, Brazil. Environ. Monit. Ass. DOI 10.1007/s10661-010-1495-3, 2010.

TRIVINHO-STRIXINO, S.; STRIXINO. G. Larvas de Chironomidae (Diptera) do Estado de São Paulo: guia de identificação de diagnose dos gêneros. São Carlos: Universidade Federal de São Carlos, 1995

WETZEL, R.G. Limnology. Florida: Saunders College Publishing. 767p, 1983

WIEDERHOLM, T. 1980. Use benthos in lake monitoring. Journal of the water pollution control federation, $52: 537-547$. 